\title{
ReMed: Jahresbericht 2016
}

ReMed, Leitungsausschuss

\section{ReMed - weiterhin grosse Nachfrage}

Die Notwendigkeit von ReMed, dem Unterstützungsnetzwerk für Ärztinnen und Ärzte in Krisen, wurde auch im vergangenen Jahr bestätigt. Der Bedarf an den ReMed-Dienstleistungen ist nach wie vor gross: Im Jahr 2016 kontaktierten knapp über 100 Personen das Unterstützungsnetzwerk. Bei den Kontaktaufnahmen, welche gemäss definiertem Ablauf innert 72 Stunden von einem ärztlichen Mitglied des Leitungsausschusses beantwortet wurden, stand in mehr als einem Drittel der Fälle die Problematik «Belastung am Arbeitsplatz» im Zentrum, gefolgt von den Problemen «Burnout» und «Depression» (je ca. ein Fünftel der Fälle).

\section{ReMed - Evaluation abgeschlossen}

Nach achtjährigem Bestehen hat eine externe Institution ReMed evaluiert, um so die Grundlage für Entscheidungen bezüglich der zukünftigen Ausgestaltung von ReMed zu schaffen. Die Resultate und die daraus resultierenden Massnahmen sind im letzten Jahr umgesetzt worden. Eine Auswahl der durchgeführten Massnahmen ist nachfolgend beschrieben.

\section{ReMed-Programm - Erfahrungen austauschen}

Im Rahmen des nationalen Programms von ReMed werden in der Deutschschweiz wie auch in der Romandie Intervisionen durchgeführt. Damit die Intervisionen in den Gesamtkontext von ReMed Schweiz eingebettet werden können, fanden im Februar und November 2016 erstmalig Erfahrungsaustausche zwischen den Erstberatenden von ReMed statt. Dieser Austausch stärkt das sichere Netzwerk von Kollegen und Kolleginnen, welche für ReMed tätig sind. Diese Treffen werden auch im laufenden Jahr fortgesetzt werden.

\section{ReMed - Projekte starten}

Im Herbst 2016 wurde durch ReMed das Projekt Coaching-Gruppen gestartet. ReMed möchte damit die Selbstreflexion, Selbstkompetenz, Gesundheitsfürsorge und Resilienz von Ärztinnen und Ärzten fördern, um Überlastung, Krisen und daraus folgende allfällige Berufsausstiege aufzufangen. Coaching-Gruppen sind individuelle Massnahmen für eine Veränderung. Das Pilotprojekt mit drei Gruppen soll erste Erfahrungen mit diesem Angebot ermöglichen.

\section{ReMed - Feedback zum Beratungsangebot}

ReMed ist bestrebt, sein Angebot kontinuierlich zu verbessern. Um diesen Prozess der Optimierung zu garantieren, haben die hilfesuchenden Ärztinnen und Ärzte neu die Möglichkeit, anonym die Qualität der Erstberatung zu bewerten. Die Erstberatenden erhalten so ein Feedback für ihren persönlichen Lernprozess und die eigene Qualitätskontrolle. Die anonymisierten und aggregierten Rückmeldungen fliessen ebenfalls in die Evaluation des Leitungsausschusses und die Weiterentwicklung des Programms ReMed ein.

\section{Angebot wirkt auch präventiv}

Im Jahr 2016 bot ReMed an diversen Kongressen und Versammlungen mit Workshops und Weiterbildungen unter Einbezug von interaktivem Theater eine Möglichkeit zur Prävention und Sensibilisierung. Die Zuschauer werden jeweils durch gezielte Moderation animiert, die Handlungsweisen der Schauspielerinnen und Schauspieler nach den eigenen Vorstellungen zu gestalten. Durch das aktive Eingreifen des Publikums wird die eigene Sicht der Dinge direkt ausprobiert und die Folgen des eigenen Handelns sind unmittelbar spürbar. Diverse Testimonials, Artikel und Interviews sind im vergangenen Jahr in der Tagespresse wie auch in der Schweizerischen Ärztezeitung erschienen. Diese zeigen, wie ReMed sich in verschiedenen Situationen verhalten und Hilfe angeboten hat.

\section{ReMed - Leitungsausschuss geändert}

Im vergangenen Jahr musste der Leitungsausschuss die Rücktritte der zwei Gründungsmitglieder von ReMed Dr. med. Michael Peltenburg und Dr. med. Jürg Bänninger zur Kenntnis nehmen. Als deren Nachfolge wurden neu Dr. med. Sabine Werner und Dr. med. Yvette Attinger Andreoli in den Ausschuss gewählt. Durch Dr. med. Y. Attinger Andreoli ist somit auch die italienische 
Schweiz erstmals im Leitungsausschuss vertreten. Die aktuelle Zusammensetzung sieht wie folgt aus:

\section{Programmleitung:}

- Dr. med. Peter Christen, Facharzt für Allgemeine Innere Medizin FMH, ärztliche Programmleitung

- Esther Kraft, lic. rer. oec., Leiterin Abteilung Daten, Demographie und Qualität FMH, administrative Programmleitung

\section{Leitungsausschuss:}

- Dr. med. Peter Birchler, Facharzt für Psychiatrie und Psychotherapie FMH

- Dr. med. Franco Gusberti, Facharzt für Psychiatrie und Psychotherapie FMH

- Dr. med. Mirjam Tanner, Fachärztin für Psychiatrie und Psychotherapie FMH

- Dr. med. Carole Weil, Fachärztin für Gynäkologie und Geburtshilfe FMH

- Dr. med. Sabine Werner, Fachärztin für Dermatologie und Venerologie FMH

- Dr. med. Yvette Attinger Andreoli, Fachärztin für Psychiatrie und Psychotherapie FMH

\section{ReMed - Bekanntheit erweitern}

ReMed

Sekretariat

Postfach 55

CH-3000 Bern 15
Rund die Hälfte der FMH-Mitglieder kennt das Angebot von ReMed. Der Leitungsausschuss möchte den
Bekanntheitsgrad des Gesamtangebotes (Krisenintervention, Begleitung, Beratung, Coaching, Mentoring, Praxispraktika etc.) zielgruppenspezifisch schweizweit steigern und wird deshalb im Jahr 2017 zielführend ein Konzept zur Erweiterung erarbeiten. Das Angebot ReMed soll unter anderem in den nachfolgend genannten Zielgruppen vermehrt bekannt werden:

- Jüngere Ärzte/Ärztinnen (unter 40 Jahren)

- Ärzte/Ärztinnen aus dem stationären Bereich

- Ärzte/Ärztinnen mit anderen Facharzttiteln als Allgemeine Innere Medizin und Psychiatrie

\section{ReMed - weitere Informationen}

Auf der Homepage www.swiss-remed.ch finden Sie interessante Erfahrungsberichte und Dokumentationen sowie wertvolle Informationen über das Unterstützungsnetzwerk.

Das nationale Programm ReMed mit dem Leitungsausschuss und den Netzwerkmitgliedern wird auch im Jahr 2017 für Ärztinnen und Ärzte in Krisensituationen da sein. Die betroffenen Ärztinnen und Ärzte können sich über die 24-Stunden-Hotline (0800 0 73633) oder per help[at]swiss-remed.ch melden.

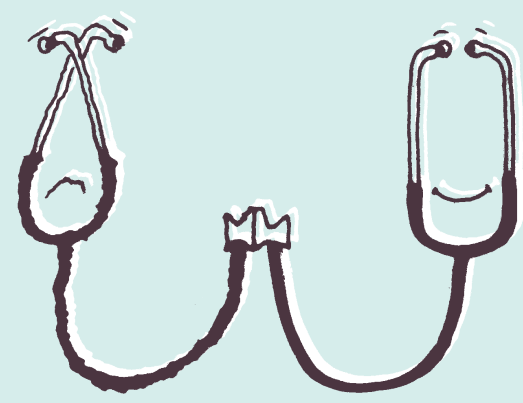

Anu
Plötzlich selbst betroffen. Krisen treffen auch Ärztinnen und Ärzte.

Lassen Sie sich helfen. Kontaktieren Sie ReMed.

24-Stunden-Hotline

$0800073633 \quad$ help@swiss-remed.ch

08000 ReMed www.swiss-remed.ch 